\title{
Resonant pumping in a multilayer impedance pump
}

\author{
L. Loumes, ${ }^{1}$ I. Avrahami, ${ }^{2}$ and M. Gharib ${ }^{1}$ \\ ${ }^{1}$ Division of Engineering and Applied Sciences, California Institute of Technology, \\ 1200 E, California Blvd., MC 205-45, Pasadena, California 91125, USA \\ ${ }^{2}$ Medical Engineering, AFEKA ý Tel Aviv Academic College of Engineering, Israel
}

(Received 22 August 2007; accepted 24 January 2008; published online 28 February 2008)

\begin{abstract}
This paper introduces the concept of multilayer impedance pump, a novel pumping mechanism inspired by the embryonic heart structure. The pump is a composite two-layer fluid-filled elastic tube featuring a thick gelatinous internal. Pumping is based on the impedance pumping mechanism. In an impedance pump, elastic waves are generated upon external periodic compressions of the elastic tube. These waves propagate along the tube's walls, reflect at the tube's extremities, and drive the flow in a preferential direction. The originality in the multilayer impedance pump design relies on the use of the thick internal gelatinous layer to amplify the elastic waves responsible for the pumping. As a consequence, only small excitations are needed to produce significant flow. This fully coupled fluid-structure interaction problem is solved for the flow and the structure using the finite element method over a relevant range of frequencies of excitation. Results show that the multilayer impedance pump is a complex system that exhibits a resonant response. Flow output and inner wall motion are maximal when the pump is actuated at the resonant frequency. The wave interaction mechanism present in an impedance pump is described here in details for the case of a multilayer impedance pump. Using energy balance for the passive portion of the elastic tube, we show that the elastic tube itself works as a pump and that at resonance maximum energy transmission between the elastic tube and the fluid occurs. Finally, the pump is especially suitable for many biomedical applications. (c) 2008 American Institute of Physics. [DOI: 10.1063/1.2856528]
\end{abstract}

\section{INTRODUCTION}

Valveless pumping in straight elastic tubes can be achieved by the use of impedance mismatch or by peristaltic motion. In a peristaltic pump, successive compressions along the tube are used to push the fluid from one tube's end to the other by positive displacement. In an impedance pump (IP), however, a single actuation location is sufficient to produce unidirectional flow, and the driving mechanism is the result of the interaction of elastic waves. These elastic waves are created by local periodic excitations of the tube at an offcenter longitudinal position. They reflect at the tube's extremities where a mismatch of impedance is present and their constructive interaction drive the flow in a preferential direction. ${ }^{1}$

Impedance represents the measure of opposition to flow presented by a system. By conventional use, impedance is resistance in the case of oscillatory systems. The term impedance is used in the electric current theory (electrical impedance), vibrating solid systems (mechanical impedance) or gas-filled systems (acoustic impedance). We are referring here to impedance as the hindrance to flow, which is expressed as the ratio of the instantaneous pressure over the instantaneous flow rate at the section considered (input impedance) ${ }^{2,3}$ In the case of the fluid-filled elastic tube system, mismatch of impedance can be practically achieved by connecting the system to tubes of different stiffnesses or different radii. A mismatch of impedance creates a wave reflection site, a necessary condition to achieve pumping

We introduce here a new kind of impedance pump that features a multilayered wall. The main advantage of this pump over a single-layer impedance pump is that it requires only small excitations to produce significant flow. Its design has been inspired from the embryonic heart whose tube geometry features a unique layered wall structure, ${ }^{4,5}$ and which is known to achieve unidirectional pumping prior to valve formation. More specifically, the embryonic heart tube's walls are made of a thick gelatin layer, the cardiac jelly, and a thinner musclelike external layer.

Following the heart tube structure, the multilayer impedance pump (MIP) presented here is modeled as a valveless composite fluid-filled elastic tube of circular cross section that is periodically excited. The bulk of the tube walls are made of a soft and thick internal gel-like layer that has the consistency of a jelly and the connectivity of a gelatin. The second external layer is a relatively stiff and thin external elastic layer. These two layers form together an efficient wave propagation system (see Fig. 1). Elastic waves are generated by periodic compressions of the elastic tube at a single off-centered location on the external surface of the tube, to reach maximum amplitude at the fluid-gelatin interface. These elastic waves, responsible for the pumping, propagate along the length of the pump and reflect at the tube's extremities. Because the flexible stiffer layer is compressible but not distensible, all large wave motion is confined inside the pump, at the fluid-gelatin interface.

Several studies of the impedance pumping mechanism have been performed on single layered thin elastic tubes of micro and macro scale using analytical, ${ }^{2,6,7}$ computational, ${ }^{1,8,9,2}$ and experimental models. ${ }^{10-14,6}$ These studies revealed a nonlinear response of the pump to the 


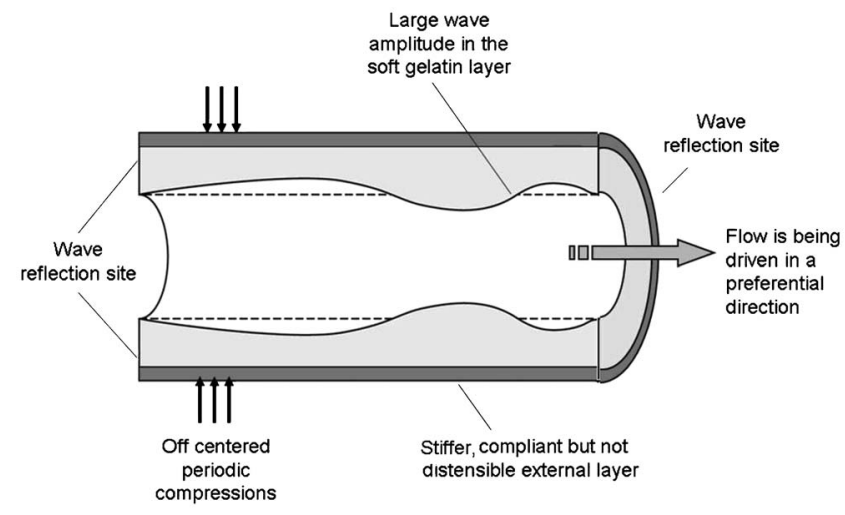

FIG. 1. Illustration of the multilayer impedance pumping mechanism.

frequency of excitation. Unlike peristaltic pumps, where pumping is proportional to the frequency of excitation, in an impedance pump, maximum exit flow is achieved when the pump is excited at its resonant frequency. The pumping performances of an impedance pump have a strong dependence on the different parameters defining the pump such as its dimensions, material properties, the pinching width, location and amplitude. In particular, experiments ${ }^{10,11}$ and numerical simulations $^{1,2}$ on a single layer IP in open and closed systems, respectively, have shown that for some frequencies of excitation, the pump exit flow is directly proportional to the actuation amplitude (up to $90 \%$ of the external radius). Therefore, to achieve significant flow, an IP must typically be compressed at relatively high amplitudes and around the resonance frequency. This strong compression can strain the tube walls, create large outward radial motion of the tube that would make the pump not compatible with confined environments, or also occlude the flow which would make the device not suitable to many biomedical applications. The proposed multilayered impedance pump addresses these difficulties with the combined use of the gelatinous and the stiffer layer. The presence of the external stiffer layer forbids large radial outward motion, while the softness and thickness of the inner gelatinous layer are used to amplify and efficiently propagate elastic waves. Therefore, in a MIP only small excitations are needed to generate elastic waves that last longer and are of greater amplitude than a single layer IP would have. In addition to its special design, the pumping is based on resonance. The pump performances are frequency dependent and maximize at resonance. One of the features of resonant pumping is that the pump requires minimal input energy to operate when exited at resonance.

To investigate the MIP response to frequency, we performed numerical simulations based on the finite element method. The choice of numerical simulations is motivated by the possibility of extensive use in design optimization and because one can isolate factors which in many cases cannot be separated experimentally. They also provide detailed descriptions of the unsteady flow field and the solving of structural behavior at any point in the elastic tube.

This paper presents the new concept of multilayer impedance pump. We will explore the dynamic response of the system to periodic excitation (fluid and structure), with an emphasis on the system's behavior at resonance conditions. Considerations on the energy exchange between the elastic tube and the fluid will highlight the concept of resonance pumping.

\section{METHODS}

\section{A. Physical model}

The pump model was a fluid-filled elastic tube with an excitation zone that is located asymmetrically with respect to the length of the pump. The pump had an aspect ratio of about 15 (see Table I and Fig. 2), and had a layered wall structure similar to the embryonic heart. The fluid domain represented about $30 \%$ of the total volume occupied by the

TABLE I. Physical parameters.

\begin{tabular}{lcc}
\hline \hline Physical parameter & Symbol & Value \\
\hline Length of the pump & $L$ & $15.2 \mathrm{~cm}$ \\
External radius of the pump & $R_{\text {ext }}$ & $1.03 \mathrm{~cm}$ \\
Fluid domain radius & $R_{f}$ & $0.55 \mathrm{~cm}$ \\
Gelatin thickness & $h_{\text {gel }}$ & $0.405 \mathrm{~cm}$ \\
Stiffer layer thickness & $h_{\mathrm{sl}}$ & $0.075 \mathrm{~cm}$ \\
Actuator location with respect to the tube's nearest extremity & $a_{l}$ & $1.2 \mathrm{~cm}$ \\
Actuator width & $a_{w}$ & $1.8 \mathrm{~cm}$ \\
Gelatin stiffness & $E_{\text {gel }}$ & $5 \times 10^{4} \mathrm{dyn} / \mathrm{cm}^{2}$ \\
Stiffer layer stiffness & $E_{\mathrm{sl}}$ & $1 \times 10^{7} \mathrm{dyn} / \mathrm{cm}^{2}$ \\
Gelatin Poisson's ratio & $\nu_{\mathrm{gel}}$ & 0.49 \\
Stiffer layer Poisson's ratio & $\nu_{\mathrm{sl}}$ & 0.3 \\
Gelatin density & $\rho_{\mathrm{gel}}$ & $1 \mathrm{~g} / \mathrm{cm}^{3}$ \\
Stiffer layer density & $\rho_{\mathrm{sl}}$ & $1 \mathrm{~g} / \mathrm{cm}^{3}$ \\
Fluid viscosity & $\mu_{f}$ & $0.01 \mathrm{~g} / \mathrm{cm} \mathrm{s}^{2}$ \\
Fluid density & $\rho_{f}$ & $1 \mathrm{~g} / \mathrm{cm}^{3}$ \\
Excitation amplitude & $A$ & $0.1 \mathrm{~cm}$ \\
Frequency & $f$ & $7 \mathrm{~Hz} \mathrm{to} 12.2 \mathrm{~Hz}$ \\
\hline \hline
\end{tabular}



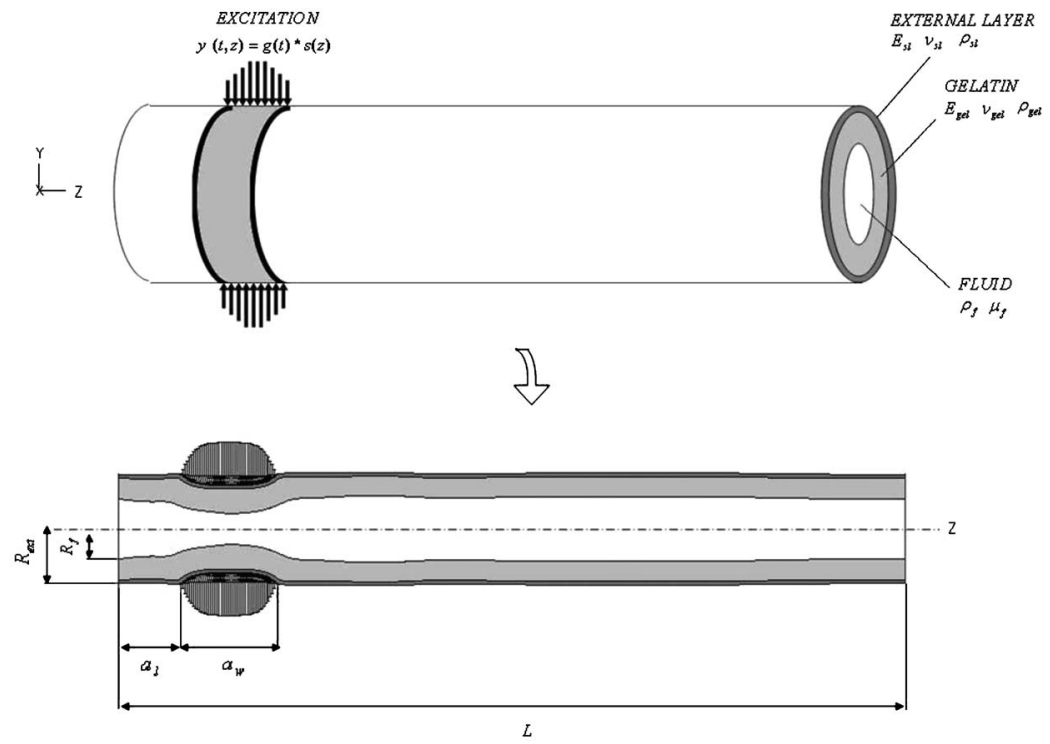

FIG. 2. (Top) 3D view of the physical model of the gelatinous impedance pump. (Bottom) 2D view in the longitudinal cross section. pump. The layered walls of the elastic tube were made of a thick gelatin layer for about $80 \%$ of the elastic tube volume, and a thin stiffer layer for the remaining 20\%. Each layer constituting the tube walls was made of an isotropic linear elastic material. The material properties of each layer have been chosen so that a large enough stiffness ratio between the elastic layers enables the combined effect of wave amplification through the gelatin and the prevention of outward motion at the external layer. In addition, following the embryonic heart structure, the stiffer layer has been given some compressibility $\left(\nu_{\mathrm{sl}}=0.3\right)$, while the gelatin was relatively incompressible $\left(\nu_{\text {gel }}=0.49\right)$.

The periodic excitation consisted of imposed radial displacements $y(t, z)$ on a section of the outer surface of the tube, Eq. (1). The pump was actuated for $20 \%$ of the period time $T$. The tube's external radius was maintained to original position during the remaining $80 \%$ of the period time. During actuation, the elastic tube was compressed following a sinusoidal time function $g(t)$ that depended on the frequency of excitation $f$, Eq. (2). The amplitude $A$ of the compression was set to $10 \%$ of the pump external radius. The spatial repartition of the compression zone followed a quadratic spatial function $s(z)$ to simulate a physical pincher, Eq. (3),

$$
\begin{aligned}
& y(t, z)=g(t) \times s(z), \quad(t, z) \in[0, T] \times\left[a_{l}, a_{l}+a_{w}\right], \\
& g(t)=A \times \sin (5 \pi f t) \times \text { Heaviside }\left(\frac{T}{5}-t\right), \quad t \in[0, T],
\end{aligned}
$$

$$
s(z)=1-\left[\frac{2\left(z-a_{l}\right)}{a_{w}}-1\right]^{4}, \quad z \in\left[a_{l}, a_{l}+a_{w}\right] .
$$

The impedance mismatch was achieved by fixing the tube's extremities, ensuring total reflection of the elastic waves. The fluid filling the tube was water.

\section{B. Mathematical model}

The fluid motion was derived by the conservative Navier-Stokes equations using the arbitrary Lagrange Eulerian formulation,

$$
\begin{aligned}
& \nabla \cdot \mathbf{v}=0 \\
& \rho_{f}\left[\frac{\partial \mathbf{v}}{\partial t}+\left(\mathbf{v}-\mathbf{v}_{g}\right) \cdot \nabla \mathbf{v}\right]+\nabla \boldsymbol{\tau}_{\mathbf{f}}=0
\end{aligned}
$$

where $\boldsymbol{\tau}_{\mathbf{f}}$ is the stress tensor, $\mathbf{v}$ is the flow velocity vector, and $\mathbf{v}_{\mathbf{g}}$ is the local coordinate velocity vector, $\rho_{f}$ is the density of the fluid, and $t$ is the time.

The fluid is Newtonian, incompressible, and viscous, and its state of stress $\boldsymbol{\tau}_{\mathrm{f}}$ follows

$$
\boldsymbol{\tau}_{\mathbf{f}}=-P \mathbf{I}+\mu_{f}\left(\nabla \mathbf{v}+\nabla \mathbf{v}^{T}\right)
$$

where $P$ is the static pressure and $\mu_{f}$ is the dynamic viscosity.

The dynamics of each layer of the flexible wall were calculated using the balance of momentum equation in Lagrangian form Eq. (7) and the constitutive relation for a linear isotropic elastic material Eq. (8),

$$
\begin{aligned}
& \nabla . \boldsymbol{\tau}_{s}+\mathbf{b}^{\mathbf{f}}=\rho \ddot{\mathbf{u}}, \\
& \boldsymbol{\tau}_{\mathbf{s}}=\lambda \operatorname{Tr}\left(\boldsymbol{\varepsilon}_{\mathbf{s}}\right) \mathbf{I}+2 \mu \boldsymbol{\varepsilon}_{\mathbf{s}},
\end{aligned}
$$

where $\boldsymbol{\tau}_{\mathbf{s}}$ is the Cauchy stress tensor, $\boldsymbol{\varepsilon}_{\mathbf{s}}$ is the strain tensor, $\mathbf{b}^{\mathbf{f}}$ is the body forces vector per unit volume, $\ddot{\mathbf{u}}$ is the acceleration vector, $\rho$ is the density, and $\lambda$ and $\mu$ are the Lamé constants of the considered structural domain.

At the fluid-structure interface the fluid was fully coupled to the gelatin. The fundamental conditions applied to the fluid-structure interface were displacement compatibility and traction equilibrium between the two surfaces,

$$
\mathbf{d}=\mathbf{u}
$$




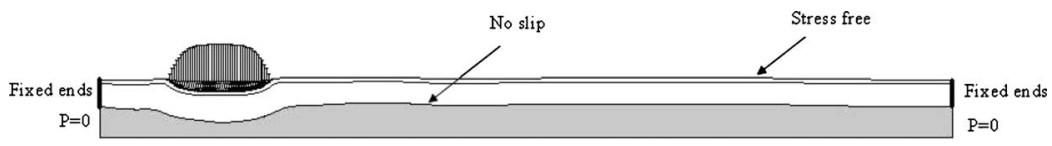

FIG. 3. Schematic of the boundary conditions.

$$
\mathbf{n} \cdot \boldsymbol{\tau}_{\mathrm{f}}=\mathbf{n} \cdot \boldsymbol{\tau}_{\mathrm{s}}
$$

where $\mathbf{d}$ and $\mathbf{u}$ are the fluid and solid displacement vectors, respectively, and $\mathbf{n}$ is the unit normal.

To ensure total wave reflection, fixed ends in both layers were modeled by imposing zero displacements in all directions and at all time at the two tube extremities,

$$
\mathbf{u}=\mathbf{0} \quad \text { at } z=0 \text { and } z=L .
$$

The no-slip and no-penetration conditions were applied at the fluid-structure interface. The tube lied in a stress-free and pressure-free environment (see Fig. 3),

$$
\begin{aligned}
& \mathbf{n} \cdot \boldsymbol{\tau}_{\mathbf{s}}=0 \quad \text { on the lateral surface of the tube, } \\
& P=0 \quad \text { at } z=0 \text { and } z=L .
\end{aligned}
$$

Initial conditions were of the resting state: Zero pressure and zero velocity in the fluid, no stress or strain in the structure.

\section{Numerical model}

The finite elements method was used to discretize both the fluid and structure domains, and the fully coupled problem was solved using the commercial package ADINA (ADINA R\&D, Inc., MA).

The fluid and the solid domain were meshed using 4-noded axisymmetric elements. The solid mesh was refined at the pinching zone. A total of 10500 elements were used, 6000 for the fluid and 4500 for the solid (see Fig. 4). An embedded actuation pincher was modeled by imposing radial displacements on a series of nodes corresponding to the pincher location, at the outer surface of the tube. The wall dynamics were solved using the small strain, small displacement hypothesis. Flow was assumed to be laminar. A constant number of 1000 time steps per pinching cycle were used to march throughout the transient simulations.

The time integration scheme was implicit Euler backward $(\alpha=1)$, which is first order accurate in time. The equations of motion were integrated by using the implicit damped Newmark scheme $(\delta=0.5, \alpha=0.25)$, and the full Newton method was used for the nonlinear equations. The fluid and solid were two-ways direct fully coupled, and the fluid mesh was updated at each time step using arbitrary Lagrange Eulerian formulation. All computations started from the resting state and are carried on until periodicity in the fluid motion was achieved. Periodicity is being reached when the mean exit flow is constant, with an error of $1 \%$, over at least 5 periods of time.

\section{RESULTS}

\section{A. Mesh and time step independence tests}

We perform the same computation for different mesh and time step refinements keeping the product $C=t_{s} \times N$ constant, where $t_{s}$ is the time step and $N$ is the total number of elements. In each test case, the model is excited at one of the highest excitation frequency $(f=11 \mathrm{~Hz})$ so that the accuracy of the simulation for lower frequencies will be ensured. Our model has a total of 10500 elements (6000 fluid elements and 4500 solid elements) and we use 1000 time step per each excitation period $(C=0.9545)$. We compare our model to four other cases (see Table II), and each computation runs until periodicity in the flow is achieved.

We define the instantaneous error in mesh refinement to be the mean error in axial velocity relative to the finest mesh at a specific point $\left(y_{o}, z_{o}\right)$, Eq. (14),

$$
\operatorname{Error}_{\left(y_{o}, z_{o}\right)}=\operatorname{mean}_{t \in\left[t_{o}, t_{o}+T\right]}\left|\frac{v_{z}^{\text {test }}\left(t, y_{o}, z_{o}\right)-v_{z}^{\text {finest }}\left(t, y_{o}, z_{o}\right)}{v_{z}^{\text {finest }}\left(t, y_{o}, z_{o}\right)}\right| \text {. }
$$

The time average of the error calculated for the point belonging to the axis of symmetry at the exit of the pump decreases with mesh refinement (see Table II) and our model has an average relative difference with the finest mesh possible $\operatorname{Error}_{(0, L)}$ of about $3 \%$.

\section{B. Identification of the natural frequencies of the system}

A free vibration test and a spectral analysis are performed. The model is impulsively actuated and the pincher is held to resting position until every motion in the fluid and solid domains disappears.

The triangular impulse duration is $1.66 \times 10^{-2} \mathrm{~s}$, corresponding to 200 time steps of $8.3 \times 10^{-5} \mathrm{~s}$ each. The time step length $t_{s}=8.3 \times 10^{-5} \mathrm{~s}$ corresponds to the smallest time step duration used throughout the computations $(f=12 \mathrm{~Hz})$.

Because the model is fairly complex, the spectral analysis of the impulse response is carried on for different parameters extracted from the flow (pressure, axial velocity) and the structure (radial displacement), and at different points throughout the model. Each of these observables \{point, parameter\} has an associated power spectrum density (PSD). Throughout the model the different observables' PSD contain the same frequencies that are expressed at different strengths (amplitude of the PSD). In order to identify the model's natural frequencies, we chose to analyze the exit flow rate varia-

$-z$

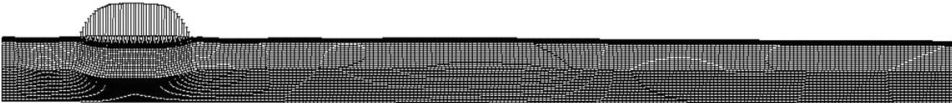

FIG. 4. 2D axisymmetric view of the mesh and pinching of imposed displacement of a series of nodes at the outer surface of the tube. 
TABLE II. The different test cases (mesh and time steps refinements) and the associated error with respect to the finest mesh.

\begin{tabular}{lccc}
\hline \hline Case No. & Number of elements & Time steps per period & Mean error \\
\hline 1 & 13650 & 1300 & 0 \\
2 & 12600 & 1200 & 0.0069 \\
3 & 10500 & 1000 & 0.0315 \\
4 & 8400 & 800 & 0.0565 \\
5 & 5200 & 500 & 0.0583 \\
\hline \hline
\end{tabular}

tion in time (see Fig. 5). Indeed, in addition to exhibiting all the natural frequencies present in the system, the exit flow rate is a relevant observable for the system pump. From the exit flow rate's PSD, we identify $f_{d}=33 \mathrm{~Hz}$ as the dominant frequency, $f_{n}=11 \mathrm{~Hz}$ as a natural frequency, and $f=22 \mathrm{~Hz}$, $f=33 \mathrm{~Hz}, f=44 \mathrm{~Hz}, f=55 \mathrm{~Hz}$ as its harmonics. Additional natural frequencies are $f=41 \mathrm{~Hz}, f=49 \mathrm{~Hz}$, and $f=59 \mathrm{~Hz}$. We studied the system around the natural frequency $\left(f_{n}=11 \mathrm{~Hz}\right)$ that was a subharmonic to the dominant frequency of the spectrum.

\section{Pulse velocity}

The pressure wave speed was calculated using a single pressure step applied at one extremity of the tube. The pressure is modeled as a normal traction force of magnitude $1 \times 10^{4} \mathrm{dyn} / \mathrm{cm}^{2}$. The time step resolution for the computation is $t_{s}=9.0909 \times 10^{-5} \mathrm{~s}$. The pressure wave speed is estimated to $172.7 \mathrm{~cm} \mathrm{~s}^{-1}$, based on the time needed for the pressure step to propagate along the model $(L=15.2 \mathrm{~cm})$ at rest. This velocity is close to the value $c_{0}=155.6 \mathrm{~cm} \mathrm{~s}^{-1}$ found using the Moens-Korteweg formula ${ }^{15}$ Eq. (15) (derived for inviscid flow in a thin walled elastic tube that possesses some material compressibility),

$$
c_{0}=\sqrt{\frac{E h}{2 \rho a\left(1-\nu^{2}\right)}},
$$

where $E$ is the stiffness of the gelatin layer $\left(E_{\text {gel }}=5\right.$ $\left.\times 10^{4} \mathrm{dyn} / \mathrm{cm}^{2}\right), h$ is the thickness of the gelatin layer $\left(h_{\text {gel }}=0.405 \mathrm{~cm}\right), \rho$ is the density of the gelatin layer $\left(\rho_{f}=1 \mathrm{~g} / \mathrm{cm}^{3}\right), a$ is the fluid domain radius $\left(R_{f}=0.55 \mathrm{~cm}\right)$, and $\nu$ is the Poisson's ratio of the gelatin $\left(\nu_{\mathrm{gel}}=0.49\right)$.
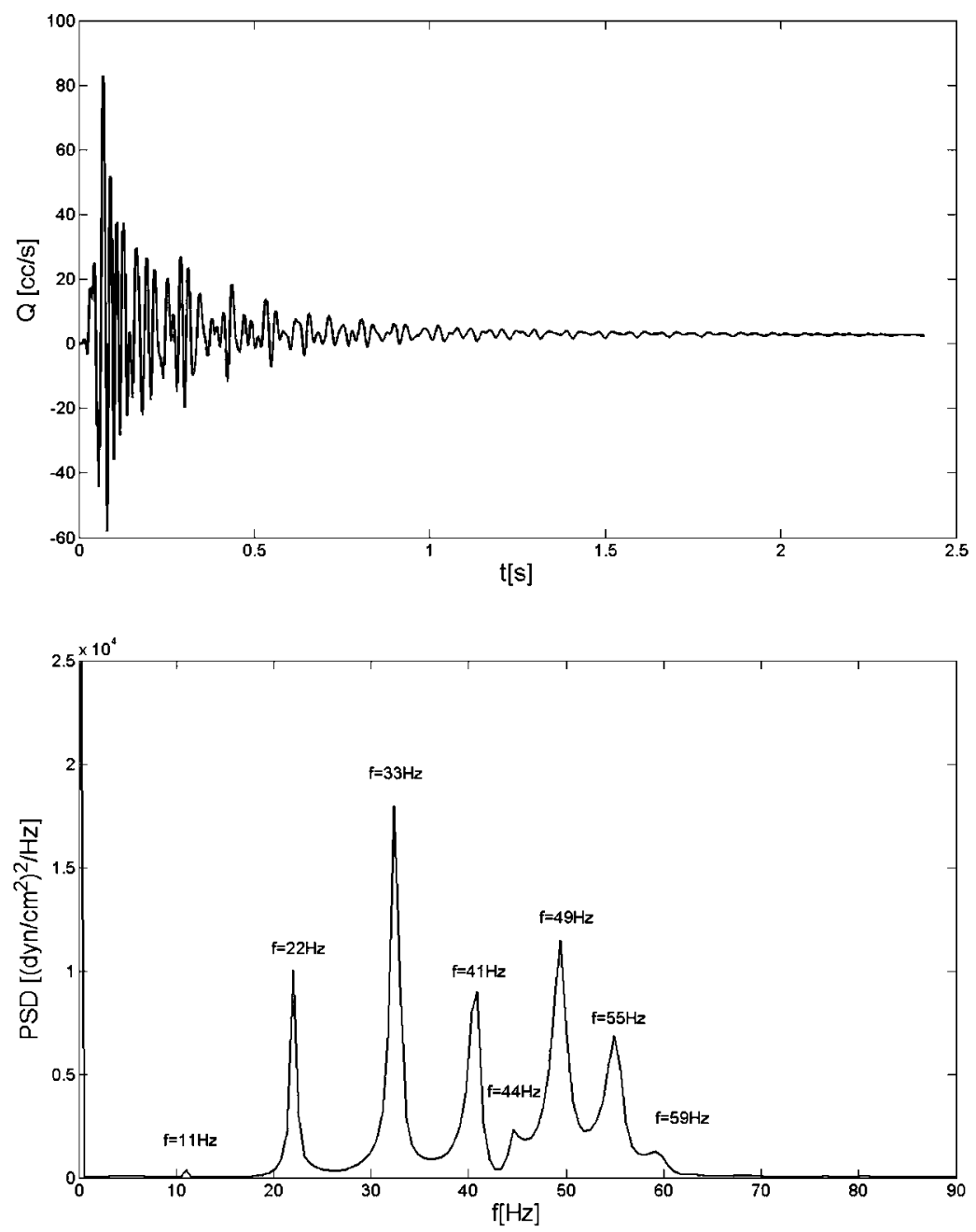

FIG. 5. Impulse response: (top) Exit flow rate variation in time under triangular impulse excitation; (bottom) the associated power spectrum density (PSD). The Fourier transform was calculated using 4096 points and a time resolution of $4 \times 10^{-4} \mathrm{~s}$. 


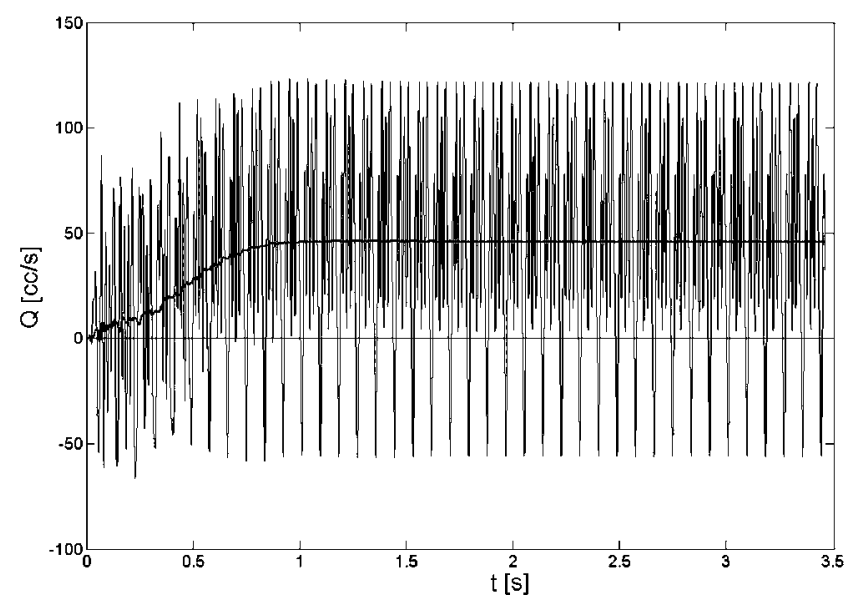

FIG. 6. Typical exit flow rate history plot. Excitation frequency is $f$ $=11.5 \mathrm{~Hz}$. Periodicity is achieved after 15 pinching cycles and mean flow at steady state is $45.7 \mathrm{~cm}^{3} / \mathrm{s}$. The solid line is a filtered curve of the flow rate using a moving average window of one cycle.

\section{Flow rate variation in time}

The instantaneous flow rate $Q\left(t, z_{o}\right)$ at a cross section located at $z=z_{o}$ of the tube is expressed as

$$
Q\left(t, z_{o}\right)=2 \pi \int_{0}^{R_{f}(t)} v_{z}\left[y\left(t, z_{o}\right), z_{o}\right] y\left(t, z_{o}\right) d y\left(t, z_{o}\right),
$$

where $v_{z}$ is the axial velocity, $y$ is the radial position, $R_{f}$ is the fluid domain radius, and $z_{o}$ is the longitudinal position of the considered cross section, and $t$ is the time. For each excitation frequency, we compute the cross-sectional flow at the pump extremity distant to the actuator $Q(t, L)$. The exit flow rate is, by convention, positive when flow is exiting the pump (flow in the $Z$ direction).

The exit flow history plots show a transient phase where the flow is building up before reaching a steady state of periodic oscillations and constant mean value (see Fig. 6).

\section{E. Mean exit flow rate and frequency}

For the different frequencies of excitation studied, the mean exit flow rate $(\bar{Q})$ is calculated by averaging at steady state conditions, the instantaneous exit flow rate $Q(t, L)$ over one period of excitation.

The mean exit flow rate is found to be nonlinearly dependent on frequency as expected for an IP. It exhibits a zone of negative flow for frequencies below $9 \mathrm{~Hz}$, and a zone of positive flow for frequencies comprised between $9 \mathrm{~Hz}$ and $12 \mathrm{~Hz}$ (see Fig. 7). Maximum positive flow reaches $86.87 \mathrm{~cm}^{3} / \mathrm{s}$ when the pump is excited at $10.1 \mathrm{~Hz}$. Therefore, for the system pump $f_{\text {res }}=10.1 \mathrm{~Hz}$ will be referred as the resonant frequency of the system. The presence of flow resonance has been also observed in a single-layer IP system. ${ }^{1,11,2}$

\section{F. Wall motion}

Each layer of the tube is of a distinct thickness and of distinct material properties which influences the speed, damping, and amplitude of the traveling elastic waves. The concept of multilayer pumping relies on large amplitude wave motion at the fluid-gelatin interface combined with a very limited motion of the external surface of the pump. The maximum wall deflection in the stiffer layer outer surface is indeed found to range from $0.37 \%$ to $6.10 \%$ from resting position, while the gelatin inner surface deflects from $24 \%$ to $32 \%$ from resting position, depending on the frequency of excitation. At resonance $f_{\text {res }}=10.1 \mathrm{~Hz}$, gelatin stretch is particularly important (see Fig. 8) and therefore plays a role in the pumping performance.

\section{G. Waves interaction in a multilayer impedance pump}

Upon compression, elastic waves are created in both layers of the tube. They travel along the length of the tube and reflect at the tube extremities. The constructive wave mechanism occurring in the tube's walls of a MIP is similar to the one described by Avrahami et al. ${ }^{1}$ for a single layer IP. When the pump is excited at resonance a strong wave interaction

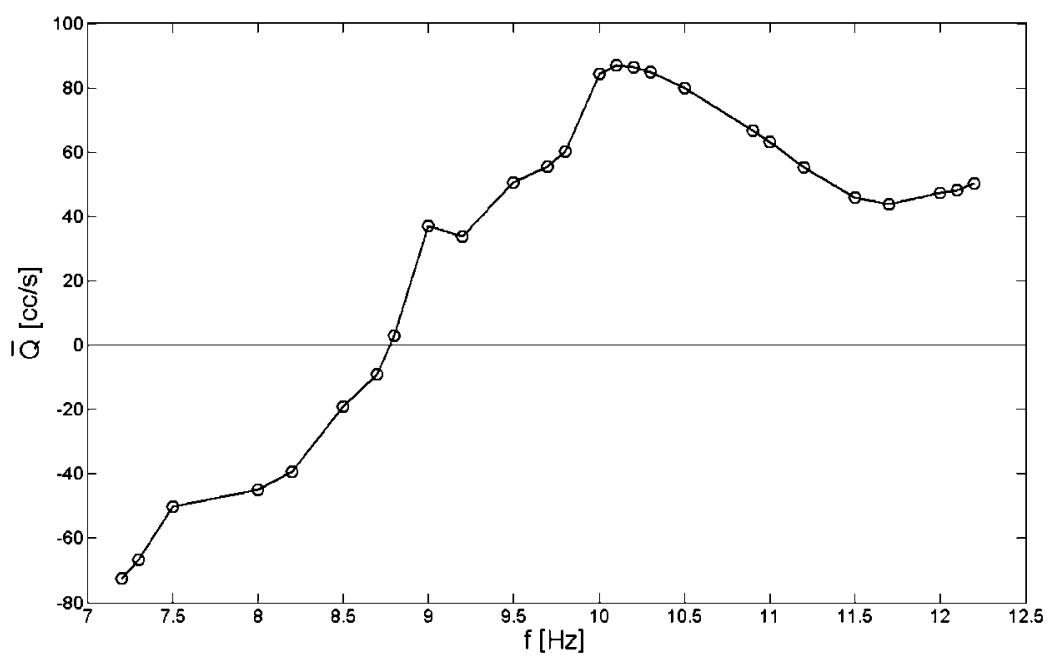

FIG. 7. Mean exit flow rate $(\bar{Q})$ as a function of the excitation frequency $(f)$. 


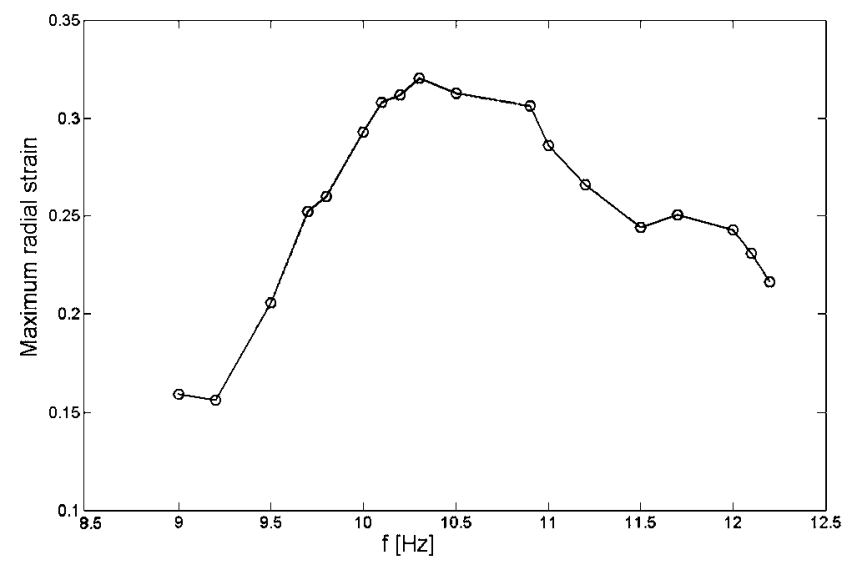

FIG. 8. Gelatin maximum positive radial strain in time and space vs frequency of excitation $(f)$.

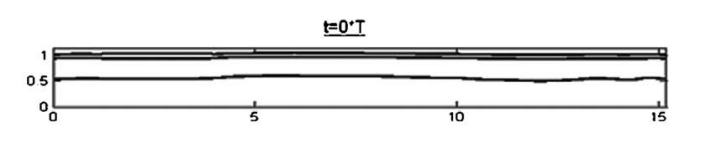

Wall position

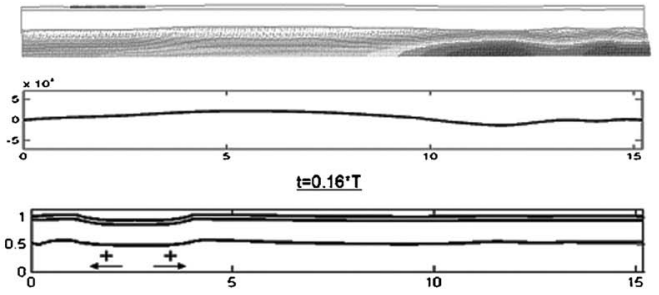

Axial velocity field

Axial pressure

Wall position
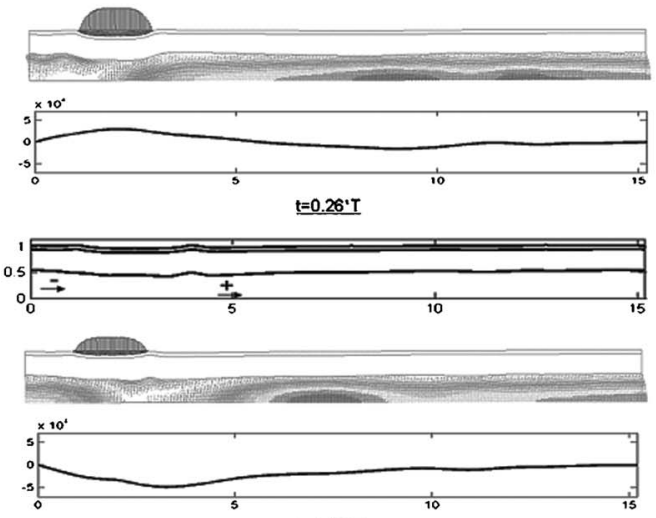

$\underline{t=0.36 \cdot T}$
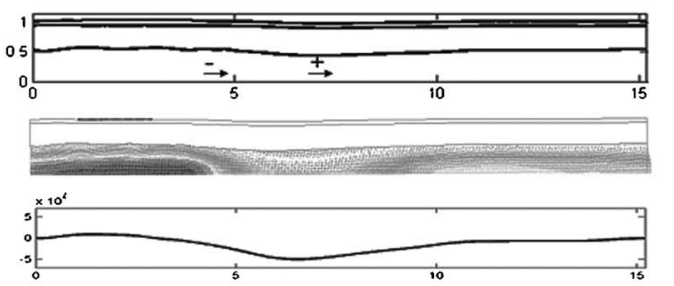

$\underline{\mathrm{t}=0.45^{\circ} \mathrm{T}}$
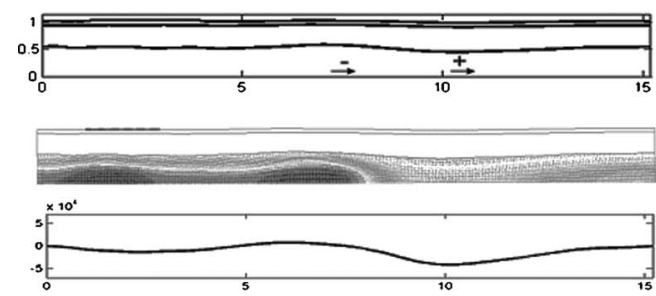

Axial velocity field

Avial pressure

Wall position

Axial velocity field

Axial pressure

Axial velocity field

Axial velocity field occurs toward the pump extremity distant from the pincher. This interaction creates a suction zone where fluid fills quickly the newly created cavity. As this suction cavity travels downstream toward the tube's extremity, a strong pressure gradient occurs between the cavity and the extremity of the tube. A net exiting flow is created by inertia.

More specifically, the wave mechanism over a period of time $T$ is as follows (see Fig. 9). The cycle begins with almost motion in the elastic tube $(t=0 \times T)$. Upon compression $(t=0.16 \times T)$, two primary positive elastic waves are created on each side of the pincher. The positive elastic wave close to the short side of the tube reflects into a negative wave $(t=0.26 \times T)$. At $t=0.36 \times T$ the pinching action is terminated and results in the creation of a reflected negative elastic wave $(z=4.8 \mathrm{~cm})$ and of a primary positive elastic

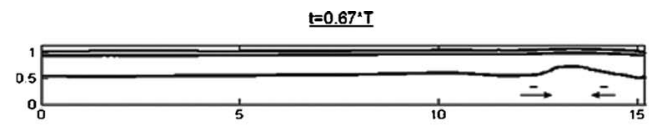

Wall position

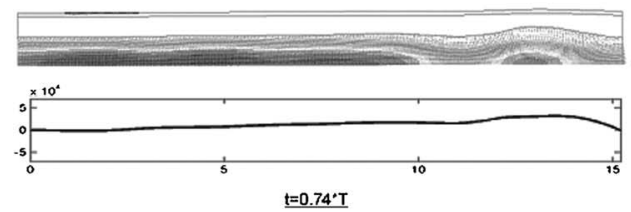

Axial velocity field

Axial pressure

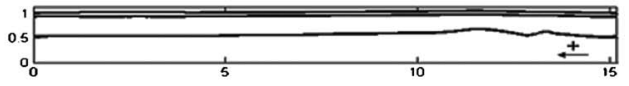

Wall position

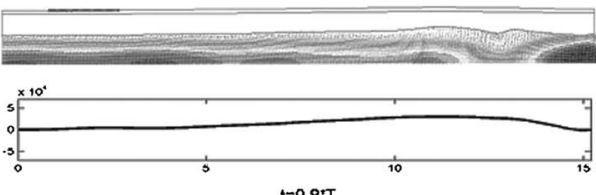

Axial velocity field

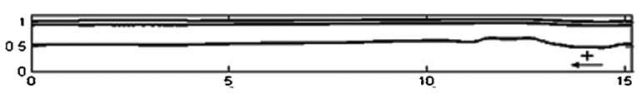

Wall position

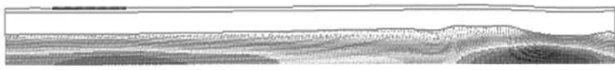

Axial velocity field

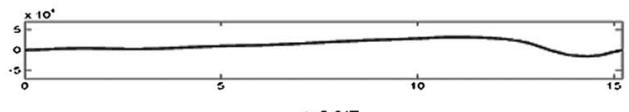

$\underline{t=0.9^{\prime} \mathrm{I}}$

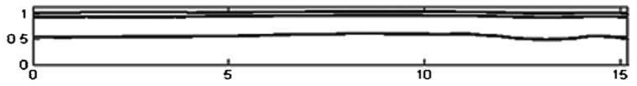

Wall position

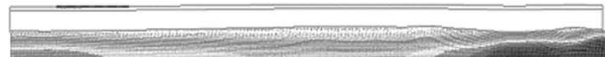

Axial velocity field

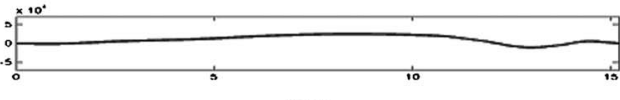

Axial pressure

$\underline{t=1 * T}$

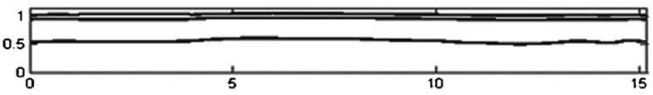

Wall position

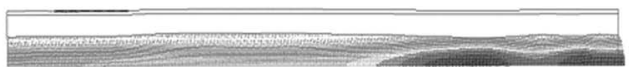

Axial velocity field

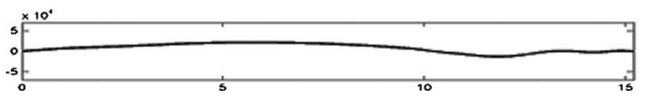

Axial pressure

FIG. 9. Illustration of the propagating waves in the multilayer impedance pump. Example for $f=10.1 \mathrm{~Hz}$. Selected frames at time $t$ as a fraction of the period time $T$. Top frame: Outline of the model, walls position against longitudinal axis. Middle frame: Corresponding snapshots of the axial velocity fluid field. Bottom frame: Axial pressure longitudinal distribution. 
wave $(z=7 \mathrm{~cm})$ traveling toward the exit of the tube. While traveling, the positive wave $(t=0.45 \times T, z=10 \mathrm{~cm})$ steepens due to the influence of the nearby forward negative wave ( $t$ $=0.45 \times T, z=7 \mathrm{~cm})$. Small amplitude secondary waves $[t$ $=0.45 \times T, z=(0,3) \mathrm{cm}]$ that have been created by the release action of the pincher and have reflected on the short side of the pincher, are now traveling toward the exit of the tube. At $t=0.57 \times T$, the forward positive wave reaches the tube's extremity, and reflects in a negative wave traveling now toward the pincher $(t=0.67 \times T)$. At that instant, a strong wave interaction occurs between this reflected wave and the still-forward-traveling negative wave creating a large suction zone $(t=0.67 \times T)$. Fluid quickly fills the newly created opening, and a strong pressure gradient is present between the cavity and the extremity of the tube as the cavity travels further downstream. Fluid is washed out by inertia and exits the pump $(t=0.74 \times T)$. The suction zone reflects at the tube extremity $(t=0.8 \times T)$, squeezing the fluid furthermore out of the pump $(t=0.9 \times T)$. Motion in the tube damps and a new cycle is about to begin $(t=1 \times T)$.

\section{H. Mechanical work done by the elastic tube}

An energy balance on the fluid inside the long portion of the elastic tube past the pincher allows us to compute the mechanical work of the elastic tube done on the fluid. Because the energy balance is made for the portion of the tube devoid of active compression (i.e., pincher), this calculation aims to focus on the energetic role of the elastic tube itself in pumping.

We use a fixed control volume (CV) delimited by the "input" and "output" cross sections, the axis of symmetry and the fluid-structure interface. The "input" cross section is located downstream next to the pinching zone at $z$ $=4.56 \mathrm{~cm}$, and the "output" upstream, just before the exit of the pump at $z=13.68 \mathrm{~cm}$, away from the exit enough to avoid the results to be biased by the "too-close" compression zone and the zero pressure boundary condition, respectively (see Fig. 10).

In the absence of added heat, the conservation of energy principle applied to the system (fluid inside the control volume) states that the time rate of change of the system total energy $(E)$ is balanced by the time rate of change of the work done to the system $(W),{ }^{16}$

$$
\frac{D E}{D t}=\frac{D W}{D t} .
$$

In the absence of gravitational forces, the work done to the system, i.e., the work done to the fluid domain, is decomposed into the work done by the environment on the fluid (involving fluid pressure and viscous terms) and the mechanical power done on the fluid and due to wall motion or shaft work $\left(W_{\text {mech }}\right)$,

$$
W=W_{\text {mech }}+W_{\text {press }}+W_{\text {viscous }} \text {. }
$$

On the other hand, the fluid's total energy per unit mass $(e)$ is decomposed to its internal and kinetic energies since gravitational forces are omitted,
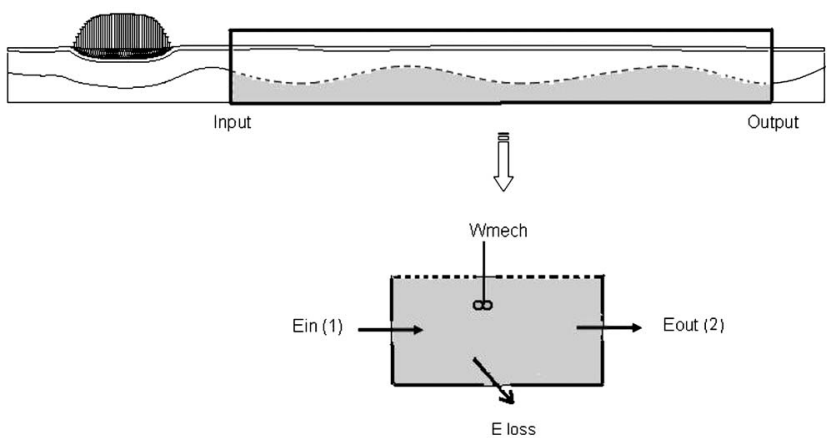

FIG. 10. (Top) Cross-sectional view of the impedance pump and the fixed control volume (solid line box) used to compute the energy balance, and the moving wall (dashed line). The fixed control volume is delimited by the input and output cross sections, the axis of symmetry, and a closure line outside the pump. The input and output cross sections delimit the passive portion of the tube for which mechanical work is calculated. We use a fixed control volume that encloses the moving wall so that to consider the wall motion as a shaft work. (Bottom) Schematic of the energy contributions to the fluid for the control volume considered. Pumping work defined as shaft work minus the losses is balanced by the differential of energy between the output $\left(E_{\text {out }}\right)$ and input $\left(E_{\text {in }}\right)$ of the system.

$$
e=e_{\text {internal }}+e_{\text {kinetic }} \text {. }
$$

Internal energy depends on temperature only, and is part of the fluid losses by internal friction $\left(\dot{E}_{\text {loss }}\right)$. Using Reynolds' transport theorem, the material derivative of the fluid's total energy $(E)$ becomes

$$
\begin{aligned}
\frac{D E}{D t}= & \frac{d}{d t} \iiint \rho e d V+\iint \rho e \mathbf{v} \cdot \mathbf{n} d S \\
= & \frac{d}{d t} \iiint \rho\left(e_{\text {internal }}+\frac{\mathbf{v}^{2}}{2}\right) d V \\
& +\iint \rho\left(e_{\text {internal }}+\frac{\mathbf{v}^{2}}{2}\right) \mathbf{v} \cdot \mathbf{n} d S \\
= & \dot{E}_{\text {loss }}+\frac{d}{d t} \iiint \rho \frac{\mathbf{v}^{2}}{2} d V+\iint \rho \frac{\mathbf{v}^{2}}{2} \mathbf{v} \cdot \mathbf{n} d S,
\end{aligned}
$$

where $d S$ is a surface differential element and $d V$ is a volume differential element of the CV.

The volume integral represents the kinetic power of the fluid inside the CV. Because of steady state periodic conditions, its contribution to the energy balance will be zero after integration over a time period. The surface integral represents the flux of kinetic energy at the $\mathrm{CV}$ boundaries, and comprises the input and output cross sections only since no fluid crosses the top part of the $\mathrm{CV}$ and $\mathbf{v} \cdot \mathbf{n}=0$ on the bottom part of the CV (axis of symmetry).

On the other hand, the rate at which the environment does work on the fluid is decomposed into fluid pressure and viscous stress components. Integration along the surfaces of the $\mathrm{CV}$ is nonzero at the input and output cross sections only, and viscous stress or shear contribution on the two cross sections is small enough to be neglected. The pressure power becomes 


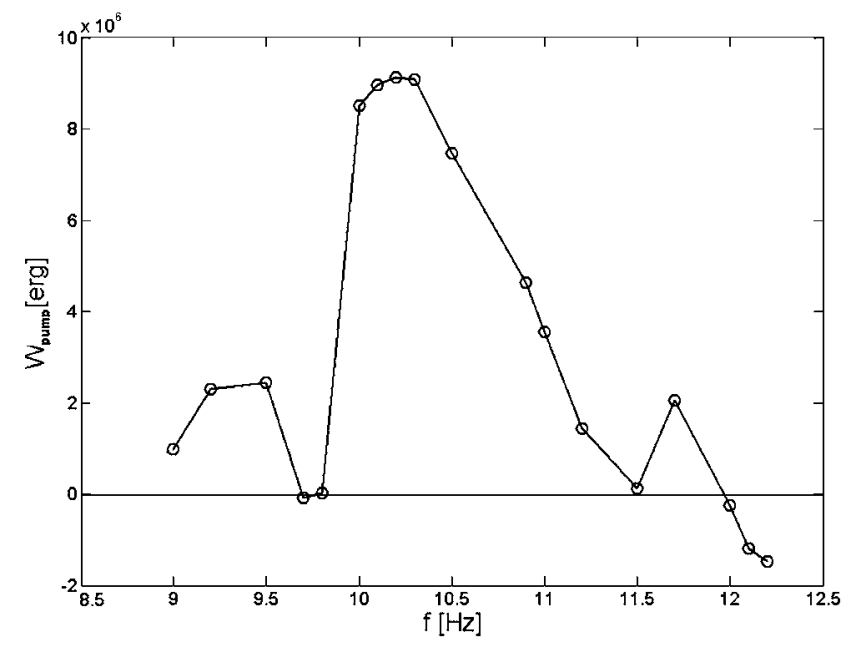

FIG. 11. Pumping work of the elastic tube $\left(W_{\text {pump }}, 1 \mathrm{erg}=1 \times 10^{-7} \mathrm{~J}\right)$ vs frequency of excitation $(f)$.

$$
\dot{W}_{\text {press }}=\iint_{1 \cup 2} P \mathbf{v} \cdot \mathbf{n} d S .
$$

Therefore, the balance of rate of change of energy is as follows:

$$
\begin{aligned}
& \dot{E}_{\mathrm{loss}}+\iint_{1 \cup 2} \rho \frac{\mathbf{v}^{2}}{2} \mathbf{v} \cdot \mathbf{n} d S=\dot{W}_{\mathrm{mech}}+\iint_{1 \cup 2} P \mathbf{v} \cdot \mathbf{n} d S, \\
& \dot{W}_{\mathrm{mech}}-\dot{E}_{\mathrm{loss}}=\iint_{1 \cup 2}\left(\rho \frac{\mathbf{v}^{2}}{2}+P\right) \mathbf{v} \cdot \mathbf{n} d S=\dot{E}_{\mathrm{out}}-\dot{E}_{\mathrm{in}} .
\end{aligned}
$$

We define the pumping power $\left(\dot{W}_{\text {pump }}\right)$ as the mechanical power done by the moving wall $\left(\dot{W}_{\text {mech }}\right)$ minus losses $\left(\dot{E}_{\text {loss }}\right)$,

$$
\dot{W}_{\text {pump }}=\dot{W}_{\text {mech }}-\dot{E}_{\text {loss }} \text {. }
$$

Finally the pumping work is found by integrating Eq. (23) over a period of time $T$,

$$
W_{\text {pump }}=\int_{t}^{t+T}\left[\iint_{1 \cup 2}\left(\rho \frac{\mathbf{v}^{2}}{2}+P\right) \mathbf{v} \cdot \mathbf{n} d S\right] d t .
$$

We found a nonlinear relationship of the pumping work and the frequency of excitation (see Fig. 11), reaching maximum around the resonant frequency. Significant positive work occurs for frequencies ranging from $10 \mathrm{~Hz}$ to $10.5 \mathrm{~Hz}$, meaning that the elastic tube does work on the fluid. It is of particular interest since the considered portion of the tube actually does not contain active components (such as pincher). This implies that the elastic tube does not act as a resistor, but contributes to pumping by transmitting energy to the fluid. At resonance the transfer of energy from the elastic tube to the flow is maximized.

\section{DISCUSSION AND CONCLUSION}

The multilayer impedance pump offers a new design for valveless pumping in confined environments where only small excitations are possible. The choice of materials for the pump model presented here is the optimized result of a previous series of combinations between elastic layers of different properties, with the scope to produce the desire wave amplification feature. The presented pump is an interesting resonant system, in which flow varies nonlinearly with frequency.

Several assumptions have been made. First, each layer constituting the elastic tube has been modeled as a linear elastic material. Dynamics of each layer has been solved using the small displacement, small strain hypothesis. Although strains in the gelatin can reach 0.3 , they are of transient nature and do not affect the linear hypothesis. The error in linearization found by performing the same simulation with the large strain assumption is less than $0.05 \%$. Second, the flow has been solved assuming laminar conditions. In pulsatile flow the onset of turbulence occurs only momentarily within the oscillatory cycle, when flow and velocity reach their peaks. ${ }^{15}$ The proposed pump model is used as a proof of concept on multilayer pumping where zones of turbulence may not influence the overall function of the pump. In that context, modeling turbulence for the transient deceleration phases in the pulsatile cycles will not bring more to the understanding of the function of the MIP. The laminar assumption may be as good as any other turbulence model, but it remains an approximation and must be considered as such.

The choice of zero pressure boundary condition at the fluid domain inlet and outlet is motivated by having a first model that would bring a proof of concept for the multilayer pumping. However, if the pump is to be implemented in the cardiovascular system, pulsatile pressure and velocity within physiological ranges have to be considered as boundary conditions at the inlet and at the outlet of the pump.

The MIP is a complex system and presents several natural frequencies. One could make an analogy between the present system (two solids coupled with a fluid), and a system of several coupled pendula, each of them having a different length. Upon excitation, each pendulum will oscillate in a manner that will exhibit a dominant frequency as well as frequencies induced by the interactions with the neighboring pendula. The system as a whole will not necessarily lock into a single resonant frequency, but will have several resonant or dominant frequencies. These dominant frequencies will be expressed at different strength depending on the pendulum (or observable) under consideration. In a similar manner, the MIP spectrum reveals several natural frequencies. For the observable considered (flow rate at the exit) the dominant frequency is $f_{d}=33 \mathrm{~Hz}$, and is a harmonic of the lowest natural frequency of the system $\left(f_{n}=11 \mathrm{~Hz}\right)$. The choice to study the pump around lower harmonics of the dominant frequency, such as the natural frequency $f_{n}=11 \mathrm{~Hz}$, has been motivated by the observation that the single layer IP had a stronger response (exit flow rate, ...) when excited around the lower harmonic of the dominant frequency. ${ }^{1,11,2}$ In addi- 
tion, with using gelatin appears a tradeoff between pinching amplitude and excitation frequency. This limits the range of frequency a specific pump model can be excited at. Due to the gelatin softness, responses to higher harmonics ( $f$ $=22 \mathrm{~Hz}, f=33 \mathrm{~Hz}, \ldots)$ or to higher natural frequencies $(f$ $=41 \mathrm{~Hz}, f=49 \mathrm{~Hz}$, and $f=59 \mathrm{~Hz})$ are possible but would require smaller pinching amplitudes.

A frequency shift of about $8 \%$ is observed between the resonant frequency $\left(f_{\text {res }}=10.1 \mathrm{~Hz}\right)$ and the natural frequency $\left(f_{n}=11 \mathrm{~Hz}\right)$. When periodically excited, the successive pinches enhance wall motion and corrupt the wave propagation. This may result in a shift between the resonant of the system $\left(f_{\text {res }}=10.1 \mathrm{~Hz}\right)$ deduced from the system response to periodic excitation and the natural frequency $\left(f_{n}=11 \mathrm{~Hz}\right)$ calculated from the impulse response spectral analysis.

We excited periodically the pump, and showed that the pump was able to produce bidirectional flow depending on the excitation frequency. The ability to reverse flow direction by adjusting the frequency of excitations has been reported in several open and closed loop experimental set ups..$^{10,11,14,6}$ In the MIP positive flow, i.e., flow exiting the pump from the extremity the farthest to the compression zone, is achieved for frequencies close to the resonant frequency $[f$ $=(9 \mathrm{~Hz}, 12 \mathrm{~Hz})]$ and reaches maximum at resonance $\left(f_{\text {res }}\right.$ $=10.1 \mathrm{~Hz})$. Negative flow is observed at frequencies below the resonant frequency $[f=(8 \mathrm{~Hz}, 9 \mathrm{~Hz})]$.

We focus on the pump response around the resonant frequency, when net mean flow is positive $[f=(9 \mathrm{~Hz}, 12 \mathrm{~Hz})]$. For that range of frequencies, the pump exhibits the largest inner wall motion. The relatively large waves at the fluid interface, however never occlude the fluid domain, and the minimum fluid radius observed throughout the computations is $R_{f}=0.37 \mathrm{~cm}$. The great gelatin stretch at resonance is to be correlated to the highest mean exit positive flow.

The wave interaction mechanism leading to pumping is similar to the one of a classic IP. ${ }^{1}$ At resonance their constructive interaction creates a suction zone toward the end of the pump that reflects and expels the fluid in a jetlike manner.

By considering the mechanical work done on the fluid by the long portion of the elastic tube past the pincher, we are able to show that the elastic tube itself acts as a pump and not as a passive resistor. For frequencies around the resonant frequency, the mechanical work is positive (tube does work on the fluid) although no active component such as a pincher is present in that portion of the tube. Upon actuation, the energy used to compress the fluid-filled elastic tube is transmitted into the elastic tube to deform it and into the fluid to move the fluid particles. The elastic tube and the fluid are exchanging energy along the tube. At a given point along the tube (characterized by Avrahami et al. ${ }^{1}$ as the velocity node), the elastic energy is given back to the fluid and contributes to the pumping. The mechanical work is clearly maximal at resonance, highlighting the concept of resonant pumping, where most efficient energy transmission between the passive elastic tube and the fluid is achieved.
The MIP can be used for different pumping applications. By tuning material properties of the elastic layers, one can pump fluids of different viscosities. The MIP has interesting features that are especially suitable for many biomedical applications as a intra-aortic assist device. ${ }^{17-19}$ It has a simple and compact design, and has no component such as blades or valves that could obstruct the flow. The multilayer structure limits all large wave motion to the fluid-gelatin interface with almost no external wall motion. Significant pumping (up to $5.16 \mathrm{~L} / \mathrm{min}$ ) is achieved for small excitation $(10 \%$ external radius) and the pump offers the possibility of bidirectional pumping, or switch, depending on the excitation frequency. The pump presented in this paper could fit the inside of an adult aorta and serve as a semipermanent intraaortic device. It could be used to enhance aortic flow in patients with congenital heart failure. Easily adaptable, the MIP could have a great use as a pediatric pump. ${ }^{20}$ It implemented on a graft for children suffering of an underdeveloped heart that have undergone a Fontan procedure. ${ }^{21,22}$ The pump can be as simple as a gelatin coated graft enliven by a pincher. Gelatin coated graft are already available, and one would only need to engineer one with a thicker gelatin layer to produce a "graft-pump." One of the advantages of the "graft-pump" is the use of the existent configuration, the mismatch of impedance being the graft connections. Other biomedical applications include polymer pumping for drug delivery at the micro- and nanoscale.

${ }^{1}$ I. Avrahami and M. Gharib, "Computational studies of resonance wave pumping in compliant tubes," J. Fluid Mech. (to be published).

${ }^{2}$ C. G. Manopoulos, D. S. Mathioulakis, and S. G. Tsangaris, "Onedimensional model of valveless pumping in a closed loop and a numerical solution," Phys. Fluids 18, 017106 (2006).

${ }^{3}$ W. R. Milnor, Hemodynamics, 2nd ed. (Williams and Wilkins, Baltimore, 1989).

${ }^{4}$ M. C. Fishman and K. R. Chien, "Fashioning the vertebrate heart: Earliest embryonic decisions," Development 124, 2099 (1997).

${ }^{5}$ A. S. Forouhar, M. Liebling, A. Hickerson, A. Moghaddam, H.-J. Tsai, J. R. Hove, S. E. Fraser, M. E. Dickinson, and M. Gharib, "The embryonic vertebrate heart tube is a dynamic suction pump," Science 312, 751 (2006).

${ }^{6} \mathrm{~J}$. T. Ottesen, "Valveless pumping in a fluid-filled closed elastic tubesystem: One-dimensional theory with experimental validation," J. Math. Biol. 46, 309 (2003).

${ }^{7} \mathrm{H}$. Thomann, "A simple pumping mechanism in a valveless tube," $\mathrm{Z}$. Angew. Math. Phys. 29, 169 (1978).

${ }^{8}$ J. S. Hansen, J. T. Ottesen, and A. Lemarchand, "Molecular dynamics simulations of valveless pumping in a closed microfluidic tube-system," Mol. Simul. 31, 963 (2005).

${ }^{9}$ E. Jung and C. Peskin, "2D simulations of valveless pumping using immersed boundary methods," SIAM J. Sci. Comput. (USA) 23, 19 (2001).

${ }^{10}$ H.-J. Bredow, "Untersuchung eines ventillosen Pumpprinzips," Fortschr.Ber. VDI, Reihe, Vol. 6, p. 1 (1968).

${ }^{11}$ A. I. Hickerson, "An experimental analysis of the characteristic behaviors of an impedance pump," Ph.D. dissertation, California Institute of Technology, 2005.

${ }^{12}$ A. I. Hickerson, D. Rinderknecht, and M. Gharib, "Experimental study of the behavior of a valveless impedance pump," Exp. Fluids 38, 534 (2005).

${ }^{13}$ A. I. Hickerson and M. Gharib, "On the resonance of a pliant tube as a mechanism for valveless pumping," J. Fluid Mech. 555, 41 (2006).

${ }^{14}$ G. Liebau, "Über ein ventilloses Pumpprinzip," Naturwiss. 41, 327 (1954).

${ }^{15}$ S. Zamir, The Physics of Pulsatile Flow (Springer, New York, 2000).

${ }^{16}$ F. M. White, Viscous Fluid Flow, 5th ed. (McGraw-Hill, New York, 2003).

${ }^{17}$ S. C. Smith, Jr., T. E. Feldman, J. W. Hirshfeld, Jr., A. K. Jacobs, M. J. Kern, S. B. King, III, D. A. Morrison, W. W. O'Neill, H. V. Schaff, P. L. Whitlow, and D. O. Williams, ACC/AHA/SCAI, "2005 Guideline update 
for percutaneous coronary intervention" (2005).

${ }^{18}$ J. Litmathe, U. Boeken, P. Feindt, R. Marktanner, and E. Gams, "Mechanical assist devices as bridging systems to transplantation: A current review, possible risks and perspectives," Transplant. Proc. 36, 3123 (2004).

${ }^{19}$ W. W. Nichols and M. F. O'Rourke, McDonald's Blood Flow in Arteries, 4th ed. (Arnold, London, 1998).

${ }^{20}$ T. J. Baldwin, H. S. Borovetz, B. W. Duncan, M. J. Gartner, R. K. Jarvik,
W. J. Weiss, and T. R. Hoke, "The national heart lung and blood institute pediatric circulatory support program," Circulation 113, 147 (2006).

${ }^{21}$ D. M. E. Bardo, D. G. Frankel, K. E. Applegate, D. J. Murphy, and R. P. Saneto, "Hypoplastic left heart syndrome," Radiographics 21, 705 (2001).

${ }^{22}$ M. D. Rodefeld, J. H. Boyd, C. D. Myers, B. J. Lalone, A. J. Bezruczko, A. W. Potter, and J. W. Brown, "Cavopulmonary assist: Circulatory support for the univentricular Fontan circulation,” Ann. Thorac. Surg. 76, 1911 (2003) 